Review began 11/27/2021 Review ended 12/08/2021 Published 12/10/2021

(c) Copyright 2021

Ram et al. This is an open access article distributed under the terms of the Creative Commons Attribution License CC-BY 4.0. which permits unrestricted use, distribution, and reproduction in any medium, provided the original author and source are credited.

\section{A Trigeminal Neuropathy From an Inactive Hepatocellular Carcinoma}

\author{
Ananth Ram ${ }^{1}$, Reji Paul ${ }^{1}$, Vineeth Viswam ${ }^{2}$, Biji Aravind ${ }^{3}$ \\ 1. Neurology, Aster Medcity, Kochi, IND 2. Otolaryngology - Head and Neck Surgery, Aster Medcity, Kochi, IND 3. \\ Pathology, Aster Medcity, Kochi, IND \\ Corresponding author: Ananth Ram, drananth.trichy@gmail.com
}

\begin{abstract}
Breast, lung, prostate, thyroid, and kidney carcinomas are the primary tumors that are known to have bony metastasis. Hepatocellular carcinoma (HCC) frequently involves the lung and lymph nodes and less commonly the osseous system. Numbness/persistent pain in the distribution of the trigeminal nerve is more likely a neuropathy. The causes are idiopathic(common), unintentional injury to the trigeminal nerve during surgery or trauma, blood vessel pressing the trigeminal nerve, tumor infiltration, multiple sclerosis, and stroke. Unresolved facial pains after conventional treatment should prompt additional investigation to rule out other causes. In this case, we report a trigeminal neuropathy of rare cause, which is a solitary metastasis from an inactive HCC involving the osseous structures.
\end{abstract}

Categories: Neurology, Pathology, Oncology

Keywords: solitary bone metastasis, trigeminal neuropathy, trigeminal neuralgia, skull base metastasis, hepatocellular carcinoma

\section{Introduction}

Breast, lung, prostate, thyroid, and kidney carcinomas are the primary tumors that are known to have bony metastasis [1]. Hepatocellular carcinoma (HCC) frequently involves the lung and lymph nodes and less commonly the osseous system. Numbness/persistent pain in the distribution of the trigeminal nerve is more likely a neuropathy. The causes are idiopathic (common), unintentional injury to the trigeminal nerve during surgery or trauma, blood vessel pressing the trigeminal nerve, tumor infiltration, multiple sclerosis, and stroke. Unresolved facial pains after conventional treatment should prompt additional investigation to rule out other causes.

\section{Case Presentation}

A 69-year-old lady, who is on treatment for diabetes, hypothyroidism, and hypertension, was detected to have HCC three years ago, a solitary space-occupying lesion in segment six of the liver. The patient underwent successful transarterial chemoembolization (TACE) and was cancer-free for the next three years.

The patient presented with a 10-day duration of right hemifacial pain that was of electric shock-like quality occurring frequently and precipitated by chewing food, wind blowing, and water splashing on the cheek, characteristic of trigeminal neuralgia. Cranial nerve examination at that time was normal. She was started on oxcarbazepine 300mg twice daily and the non-contrast MRI of the Brain was reported to be normal. Two weeks later, she presented with worsening hemifacial pain and doubling of vision. She described the pain as burning, associated with tinnitus, numbness of the right maxillary and mandibular divisions of the trigeminal nerve. Clinical examination revealed a right lateral rectus palsy, absent corneal reflex, and reduced pin sensation of the V2-V3 divisions of the right trigeminal nerve. The remainder of her neurological examination was normal.

Given the above findings, an alternate diagnosis was suspected. A contrast MRI brain (Figures $1 \mathrm{~A}-1 \mathrm{C}$ ) revealed a $2.9 \times 2.4 \mathrm{~cm}$ destructive solid heterogeneously enhancing mass centered in the medial portion of the right middle skull base involving the greater wing of sphenoid, lateral sphenoid body, and pterygoid plates extending into the intracranial portion and infratemporal fossa. There was bony erosion of the petrous segment and cavernous portion of the carotid canal with narrowing of the petrous and posterior cavernous portion of the right internal carotid artery and invasion of the right lateral sphenoid body and petrous apex involving the right abducens nerve. 


\section{Cureus}

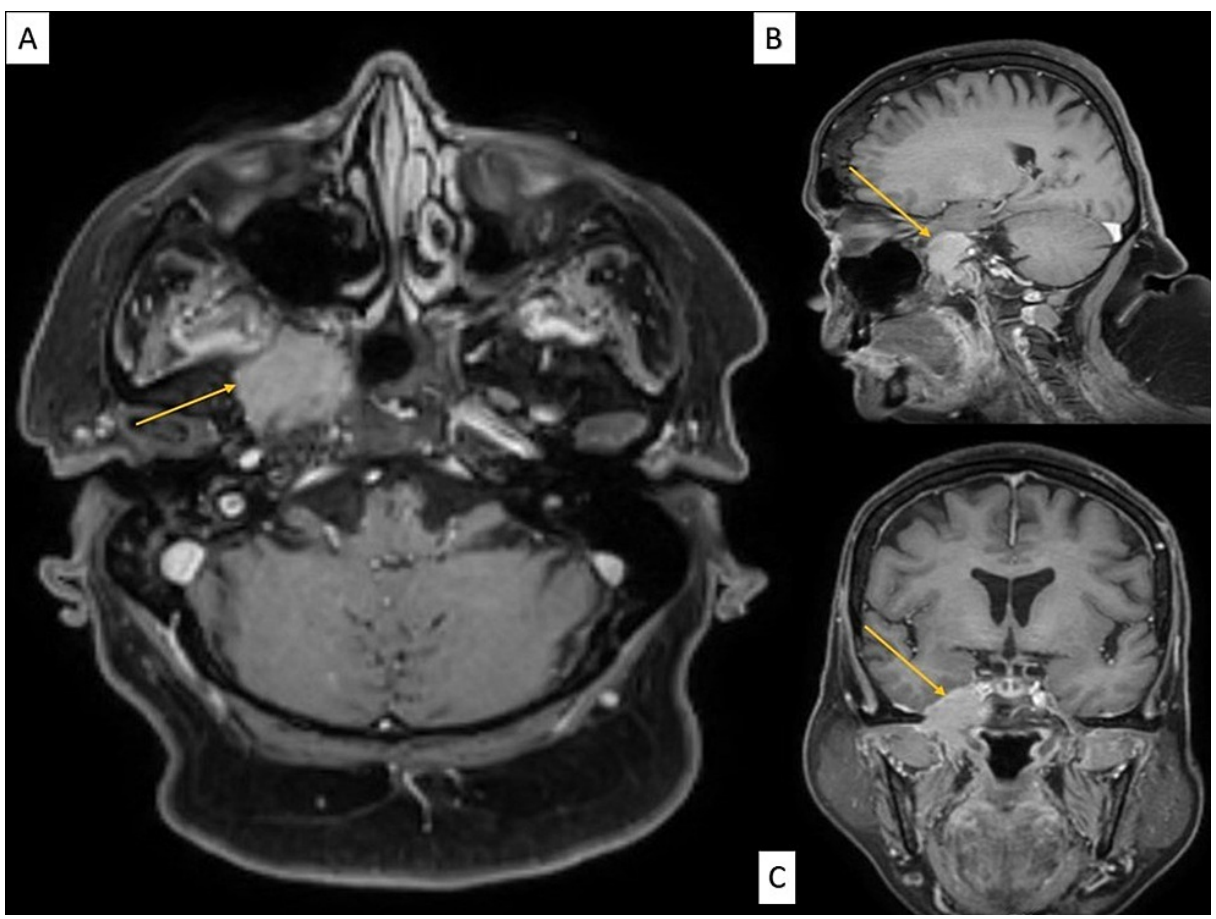

FIGURE 1: (A) Axial view, (B) sagittal view, and (C) coronal view of the MRI of the patient demonstrating a destructive solid heterogeneously enhancing mass of $2.9 \times 2.4 \mathrm{~cm}$ (yellow arrow) centered in the medial portion of the right middle skull base involving the greater wing of sphenoid, lateral sphenoid body, and pterygoid plates extending into the intracranial portion and infratemporal fossa.

Diagnostic considerations included metastasis, primary aggressive lesion/malignancy involving the middle skull base. Hence she underwent whole-body PET CT (Figures 2A-2C), which showed mildly enhancing right skull base lesion as noted in the MRI, which appears to wash off in the delayed sequences, suggesting a possibility of inflammatory vs neoplastic lesion. No FDG avid suspicious lesions were noted at the post-TACE site in segment six of the liver or the remaining liver parenchyma. 


\section{Cureus}

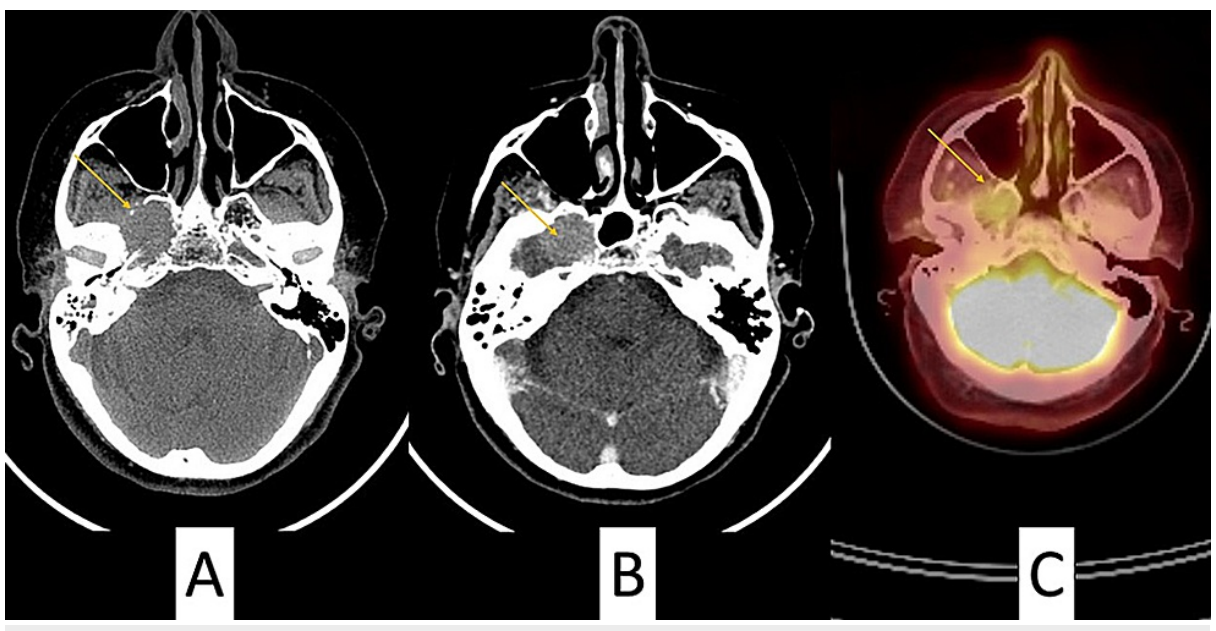

FIGURE 2: (A, B) Destructive solid heterogeneously enhancing mass of $2.9 \times 2.4 \mathrm{~cm}$ (yellow arrow) centered in the medial portion of the right middle skull base involving greater wing of sphenoid, lateral sphenoid body, and pterygoid plates causing bony erosion of petrous segment. (C) PET CT showing mildly enhancing right skull base lesion in the same area as the location of the mass.

The patient underwent an endoscopic skull base biopsy. Via a transnasal approach, the mass of $2.9 \times 2.4 \times 3.4$ $\mathrm{cm}$ was located just lateral to the paraclival and petrous portion of the carotid artery. The entire mass and a small fungal ball in the posterior ethmoid sinus were removed.

The histopathological analysis showed fragments of a neoplasm composed of trabeculae and glands lined by cells with eosinophilic nuclei and pleomorphic nuclei with nucleoli and occasional inclusions.

Immunohistochemistry analysis (Figures 3A-3E) showed strong positivity for glycipan 3 and patchy positivity for hepar and cytokeratin and negative for CK7, CK20, GATA 3, PAX8, TTF1, S100, and EMA suggesting metastasis from HCC. 


\section{Cureus}

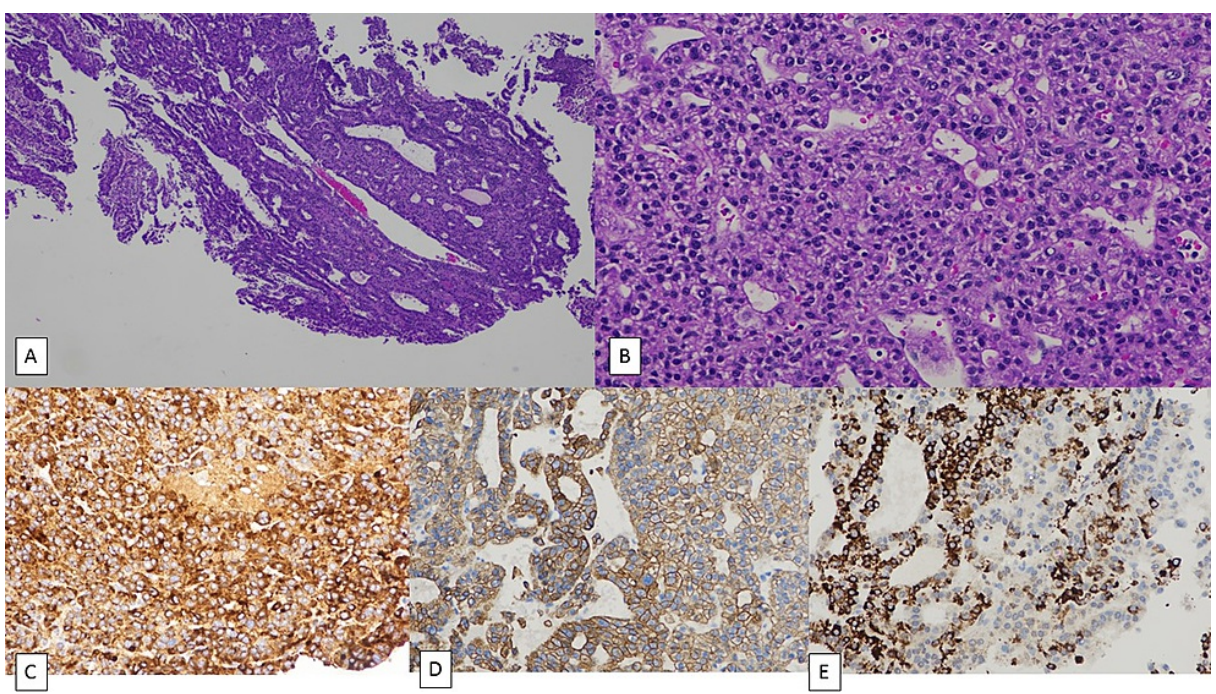

FIGURE 3: (A) Fragments of neoplasm with cells arranged as cords and trabeculae, H\&E x 40. (B) The neoplastic cells show eosinophilic cytoplasm and nuclei with mild pleomorphism, H\&E x 200. (C) IHC for glypican showing strong diffuse positivity $x$ 200. (D) IHC for cytokeratin showing diffuse positivity $\times 200$. (E) IHC for hepar showing patchy positivity $\times 200$.

IHC - Immunohistochemistry

The patient improved symptomatically and is currently symptom-free.

\section{Discussion}

According to the International Classification of Headache Disorders, typical trigeminal neuralgia is characterized by recurrent paroxysms of unilateral pain in the distribution of one or more divisions of the trigeminal nerve, which does not radiate beyond the distribution, lasts for seconds to two minutes, the severe intensity with an electric shock-like quality and precipitated by innocuous stimuli [2]. This was consistent with our patient. Our patient had involvement of V2-3 divisions of the right trigeminal nerve and right sixth nerve.

The differential diagnosis to consider in the setting of multiple cranial nerve palsy in the background of HCC are meningeal carcinomatosis, meningeal infections, and skull base metastasis, although radiological and spinal fluid analysis would help in differentiating most cases.

Incidence of HCC metastasis accounts for about $5 \%$, most often involving the lungs and the regional lymph nodes [3]. Involvement of the osseous structures is seen in about $2 \%$ to $16 \%$, which are the vertebrae, ribs, pelvic, and long bones [4]. Metastasis to the skull base is reported to be $0.4 \%-1.6 \%$. Solitary metastasis to the skull base without symptomatic evidence of primary in the liver or other sites is an extremely rare and uncommon presentation of HCC [5].

Hsieh et al. [6] found 68 patients with skull metastasis from HCC. The most common presentation was a subcutaneous liver mass with occasional painful sensations, followed by cranial nerve deficits, such as dysphagia, deafness, visual disturbances, facial numbness, of which the latter two symptoms were noted in our patient. Rad et al. reported a solitary cavernous sinus metastasis from HCC without metastasis to other sites in a patient who presented with acute painful ophthalmoplegia. Similar presentations have been reported by Goto et al. [7] and Kato et al. [8].

Trivedi et al. [9] reported a patient with an isolated skull base metastasis as the first manifestation of HCC, who presented with right 3,4,6 cranial nerve palsy and upon evaluation was noted to have HCC.

\section{Conclusions}

The uniqueness about our patient is that she had a cancer-free liver and presented with symptoms suggestive of trigeminal neuropathy. Our patient neither had a liver dysfunction at presentation nor at any time during her admission. 
Unresolved trigeminal neuralgia despite medical management, needs detailed evaluation and skull base metastasis from tumors, isolated or not, should be ruled out. Early investigation and management improve patients' quality of life.

\section{Additional Information \\ Disclosures}

Human subjects: Consent was obtained or waived by all participants in this study. Conflicts of interest: In compliance with the ICMJE uniform disclosure form, all authors declare the following: Payment/services info: All authors have declared that no financial support was received from any organization for the submitted work. Financial relationships: All authors have declared that they have no financial relationships at present or within the previous three years with any organizations that might have an interest in the submitted work. Other relationships: All authors have declared that there are no other relationships or activities that could appear to have influenced the submitted work.

\section{References}

1. Fukutomi M, Yokota M, Chuman H, et al.: Increased incidence of bone metastases in hepatocellular carcinoma. Eur J Gastroenterol Hepatol. 2001, 13:1083-8. 10.1097/00042737-200109000-00015

2. Arnold M: Headache classification committee of the international headache society (IHS) the international classification of headache disorders. Cephalalgia. 2018, 38:1-211. 10.1177/0333102417738202

3. Murakami R, Korogi Y, Sakamoto Y, et al.: Skull metastasis from hepatocellular carcinoma: CT, MR and angiographic findings. Acta Radiologica. 19951, 36:597-602. 10.3109/02841859509176756

4. Kim SR, Kanda F, Kobessho H, Sugimoto K, Matsuoka T, Kudo M, Hayashi Y: Hepatocellular carcinoma metastasizing to the skull base involving multiple cranial nerves. World J Gastroenterol. 2006, 12:6727-9. 10.3748/wjg.v12.i41.6727

5. Rad MF, Rad EF, Mashadinezhad H, Omidi A, Rahrooh M: Single cavernous sinus metastasis as the first manifestation of hepatocellular carcinoma-a rare case report and review of literature. Neurosurg Quarterly. 2010, 20:203-7. 10.1097/WNQ.0b013e3181eb26e7

6. Hsieh CT, Sun JM, Tsai WC, Tsai TH, Chiang YH, Liu MY: Skull metastasis from hepatocellular carcinoma . Acta Neurochir (Wien). 2007, 149:185-90. 10.1007/s00701-006-1071-3

7. Goto T, Dohmen T, Miura K, et al.: Skull metastasis from hepatocellular carcinoma with chronic hepatitis B . World J Gastrointest Oncol. 2010, 2:165-8. 10.4251/wigo.v2.i3.165

8. Kato H, Kanematsu M, Goshima S, et al.: Skull base metastasis from hepatocellular carcinoma revealed by cranial nerve palsy: reports of two cases. Eur J Radiol Extra. 2005, 1:1-4. 10.1016/j.ejrex.2005.03.013

9. Trivedi P, Gupta A, Pasricha S, Agrawal G, Shah M: Isolated skull base metastasis as the first manifestation of hepatocellular carcinoma--a rare case report with review of literature. J Gastrointest Cancer. 2009, 40:104. 10.1007/s12029-009-9081-z 